\title{
Un expediente sobre la labra de moneda de oro en México en el Archivo General de Indias
}

File on the Minting of Gold Coins in Mexico in the Archivo General de Indias

\section{Pedro Damián Cano Borrego}

Doctorando en Historia y Arqueología

Universidad Complutense, Madrid

pietroyanaky@telefonica.net

\section{Resumen}

El presente artículo estudia un expediente conservado en el Archivo General de Indias, con signatura AGI, MÉXICO, 77, R.3, N.50, relativo a la petición realizada por el virrey conde de Alba de Liste para que se autorizase la labra de moneda de oro en la ceca de la capital virreinal. Esta petición, contestada por Real Cédula, no fue atendida hasta ocho años después, debido principalmente al celo de Francisco de Montemayor de Cuenca, oidor de la Real Audiencia de México, a pesar de la resistencia de algunos de los principales mercaderes de la ciudad.

Palabras clave: moneda, oro, consulado de México, Casa de Moneda

\section{Abstract}

This article studies a file preserved in the Archivo General de Indias, with title "A.G.I., MEXICO, 77, R.3, N.50", concerning the petition of the viceroy Conde de Alba de Liste to authorize the minting of gold coins in the mint of the viceroyal capital. This request, answered by the Real Cedula, was not dealt with until eight years later, mainly due to the zeal of Francisco de Montemayor de Cuenca, judge of the Real Audiencia of Mexico, in spite of the resistance of some of the main merchants of the city.

Keywords: Currency, gold, Mexican Consulate, Mexican Mint 
La historia monetaria moderna en Nueva España comienza, según Antonio Beltrán, con una posible acuñación llevada a cabo en Tenochtitlán por Hernán Cortés, si bien es posible que se tratase realmente de lingotes de oro marcados con el punzón real. ${ }^{1}$ James Carson, por su parte, reproducía en su obra de 1885 una moneda de cobre de 55 granos, no citada por Alois Heiss en su obra de referencia de 1865, que sería la más antigua de las emisiones del Nuevo Mundo. ${ }^{2}$ Esta pieza, que precede a las emisiones regulares en los virreinatos indianos y que probablemente no fue grabada por un tallador real, fue relacionada por Carson con la emisión antes citada. Bernardino Fernández de Tapia acusó a Cortés de haber batido moneda en 1522, aunque probablemente se trató de la conversión del oro en polvo en discos en los que se marcaron los pesos.

Ya en 1521 se había instalado en Coyoacán un taller de fundición, donde se fundieron más de ciento treinta mil castellanos, y en 1526 el visitador Luis Ponce de León, a su llegada a Nueva España, trajo nuevos cuños para el marcado del oro y la plata y un mandato expreso de estudiar la conveniencia de instalar en el territorio una Casa de Moneda. Néstor Ricardo Chacón afirma que las primeras monedas acuñadas lo fueron en plata, en 1522, y que si bien en 1526 se envió a México un cuño con la divisa plus ultra, no llegó a usarse. ${ }^{3}$

La primera moneda áurea hispánica conocida en Nueva España fue el peso de oro de Tepuzque.4 "Tepuzque" sería la versión castellanizada de la palabra Teputzli, cobre en lengua náhuatl. Los castellanos llamaron guanín al oro bajo de ley, al estar aleado con cobre en proporciones cercanas a 50\%, que recibía los nombres de tuob entre los taínos y caracoli entre los caribes. El cronista Bernal Díaz del Castillo da testimonio de su existencia. ${ }^{5}$ También se utilizaron las conocidas como águilas de Moctezuma o cuauhtli, reservadas para pagos de importancia.

\footnotetext{
${ }^{1}$ Beltrán Martínez, Introducción a la numismática universal, p. 411.

${ }^{2}$ Carson Brevoort, Early Spanish \& Portuguese Coinage in America, p. 14.

${ }^{3}$ Chacón, Derecho monetario, pp. 63-64.

${ }^{4}$ Gil Farrés, Historia de la moneda española, p. 411.

${ }^{5}$ Vázquez Pando, "Algunas observaciones sobre el derecho monetario de la Nueva España”, pp. 1685-1686.
} 
Los pesos de Tepuzque no tuvieron un valor uniforme, sino que variaba según su peso y ley. Normalmente contenían, como su propio nombre indica, aleación de cobre, y en 1536 se fijó su paridad con la moneda de cuenta en 272 maravedíes, lo que suponía una ley de 13.6 quilates. Con ello el tomín de oro equivalía al real de plata castellano, con un valor de 34 maravedíes. El Cabildo de México intentó que se batiesen piezas de esta especie con valores de uno, dos y cuatro tomines de oro. ${ }^{6}$ El virrey Mendoza, por Ordenanza de 15 de junio de 1536, estableció que su valoración se fijase en ocho reales, al fijar, asimismo, el valor de un tomín de oro de Tepuzque en un real, dándole con ello un valor cierto y constante, y para Pradeau su circulación duró desde 1522 hasta el reinado de Felipe II, si bien a partir de 1536 se recogieron para recuperar el oro y el cobre que contenían.

En cuanto al cobre, Fabián de Fonseca recogía un auto de Antonio de Mendoza de 9 de febrero de 1546, previniendo al corregidor de Michoacán y a otros de rescatar el cobre que se consideraba necesario para la labor de la moneda de vellón que se había mandado sellar, a cargo de lo obtenido con los tributos y a razón de 18 pesos de tripus por cada quintal conducido y entregado en la ciudad. ${ }^{7}$

Como es lógico, la acuñación de moneda en suelo indiano suponía que la misma tendría que tener su valor ajustado al de Castilla, ${ }^{8}$ por lo que la diferencia anteriormente dicha habría de desaparecer. En este sentido, la Real Cédula de 11 de mayo de 1535 antes citada, contenida en la Recopilación publicada en el reinado de Carlos in como Ley Primera del título referente a las Casas de Moneda y sus Oficiales, establecía la subsidiariedad del

\footnotetext{
${ }^{6}$ Céspedes del Castillo, "Las cecas indianas en 1536-1825", p. 50.

${ }^{7}$ Fonseca y Urrutia, Historia general de la Real Hacienda, p. 115.

${ }^{8}$ Recopilación de las Leyes de las Indias (en adelante RLI), libro IV, título XXIII, ley IX, Que la moneda de plata sea del mismo valor, peso, y cuño, que la de estos Reynos de Castilla, Carlos I, Valladolid, 10 de mayo de 1544; Fonseca y Urrutia, Historia general de la Real Hacienda, p. 115.
} 
derecho castellano en todo lo no prevenido especialmente en ese título. $^{9}$

A ello contribuyó que el valor de la moneda de ocho reales de plata, 272 maravedíes, se correspondía con el citado para el peso de Tepuzque. Es por ello por lo que cuando se comenzó a acuñar se la comenzó a denominar peso. ${ }^{10}$ En la Casa de Moneda de México se acuñaron piezas de plata y vellón, quedando la labor del oro reservada a la Casa de Sevilla, aunque en la práctica la actividad se redujo al numerario de plata, debido al rechazo popular a las emisiones de vellón.

La unidad de peso para metales preciosos fue, al igual que en Castilla, el marco, alrededor de 230 gramos. En el caso de las piezas de oro, que no se emitieron hasta el siglo siguiente, sus divisores eran el castellano, el tomín y el grano, que se utilizaron como moneda corriente. En cuanto a la plata aparecían la onza, equivalente a 1/8 de marco o 28.75 gramos, el tomín de plata, de 1/384 o 0.559 gramos, y el grano de plata, de 1/4608 de marco o 0.049 gramos.

El conde de Moretti definía, en 1828, el castellano como una moneda de oro española del siglo xiv con el valor de la sexta parte de una onza de oro, con un valor de 14 reales y 14 maravedíes de plata. Como unidad de peso, se correspondía a la cincuentésima parte de un marco, dividida en ocho tomines, y compuesta de 24 quilates, 96 granos o 768 partes del grano. ${ }^{11}$

La labra de moneda de oro y vellón había sido prohibida en $1565,{ }^{12}$ en tiempos de Felipe II, para las cecas indianas, salvo en aquellos casos en que fuese permitido. Según Gil Farrés, la primera emisión áurea oficial se llevó a cabo en Santa Fe del Nuevo Reino, donde en 1622 se acuñaron 70 marcos de oro, y los primeros ejemplares que se

\footnotetext{
9 “...y todos guarden las leyes de las Casas de moneda de estos Reynos de Castilla, que tratan de la labor del oro, y plata en lo que no estuviere dispuesto especialmente por las leyes de este título", RLI, libro IV, título xxiII, ley I.

${ }^{10}$ Villaplana Persiva, Historia del Real de a Ocho, p. 70.

${ }^{11}$ Moretti, Manual alfabético, p. 30.

${ }^{12}$ Mandamos, Que en las Indias se labre moneda de plata, y no de oro, ni vellon, si no estuviese permitido, ó se permitiere por Nos, RLI, libro IV, título XXIII, ley III.
} 
conservan son acuñaciones de doblones de 1627 del Nuevo Reino. La primera onza de ocho escudos, según este autor, se acuñó en Lima en 1659, aunque algunos autores la consideran un ensayo. Según Alan K. Craig, la moneda áurea batida en Lima destaca por la calidad de los motivos grabados y la redondez de los siempre finos cospeles en la que fue acuñada. ${ }^{13}$ Estas últimas emisiones se llevaron a cabo siendo virrey del Perú el conde de Alba de Liste, impulsor, como veremos, de la emisión de monedas áureas en el virreinato septentrional, y que son conocidas con el nombre de Estrella de Lima.

\section{La petición del virrey conde de Alba de Liste}

El "Expediente sobre la conveniencia de labrar moneda de oro en la Casa de la Moneda de Mexico", consta de un conjunto de manuscritos recopilados, y numerados con letras de la "a" a la "g". El código de referencia del Archivo General de Indias es ES.41091.AGI/23.10.77 y la signatura MÉXICO,77,R.3,N.50, unidad dentro de las Secciones de Gobierno, Audiencia de México, Cartas y expedientes del presidente y oidores y Cartas de Audiencia. La fecha de formación de la misma fue el 30 de marzo de 1653, y su finalización el 4 de noviembre de 1664. Se trata de una unidad documental compuesta, en cuyos índices de descripción se incluyen la Casa de Moneda de México, Casa de moneda y Oro. Se encuentra digitalizada y a disposición de quien quiera consultarla en el Portal de Archivos Españoles.

El primero de los documentos incluidos en el expediente es la proposición realizada por Luis Enríquez de Guzmán, conde de Alba de Liste y vigésimo primer virrey de Nueva España, en fecha 30 de marzo de 1653, cuando ya había sido promovido al cargo de virrey del Perú, de que se permitiese la labra de moneda de oro en México. ${ }^{14}$

La misma, redactada en dos hojas, comienza con una invocatio representada de forma simbólica, mediante una pequeña cruz, a la

${ }^{13}$ Craig, Spanish colonial gold coins in the Florida Collection, p. 20.

${ }^{14}$ Archivo General de Indias (en adelante AGI), MÉXICO, 77, R.3, N.50. 
manera habitual de las peticiones realizadas a la Corona. No se encuentran en esta proposición expresamente ni una intitulatio donde el emisor del mismo se identifique mediante su nombre o cargo ni una directio o identificación del destinatario, y la salutatio se reduce a la palabra Señor situada justo debajo de la cruz. Al margen, encontramos el dígito 31 subrayado y la razón de la misiva, "Proponiendo las raçones que ay para que S.Mag se sirva de permitir que se labre moneda de oro en Mexico".

En el cuerpo del documento, no muy extenso, el virrey da cuenta al monarca de los motivos de su petición. Comienza con una notificatio en la que explica que "Haviendo reparado que en la Casa de la moneda de esta Ciudad no se labra oro, y deseando saver que causas del Servicio de S Mag ${ }^{\mathrm{d}}$ podian dar motivo a ello". Tras la misma viene la expositio, recogiendo las razones y antecedentes que dan lugar a su proposición. En la misma, el virrey hace referencia, en primer lugar, a la Instrucción remitida al virrey don Antonio de Mendoza por el emperador Carlos v, de la que envió testimonio incluso, por la que se le ordenaba la acuñación de moneda de plata y vellón guardando las leyes de las Casas de Moneda dadas por los reyes católicos, ${ }^{15}$ y en la que igualmente se recogía que de momento no debía de labrarse moneda de oro.

El virrey consideraba que si no hubiese otras razones superiores convendría que el oro se labrase en la ceca de México. Ello supondría, según el conde, el incremento del señoreaje o derecho de amonedación. Los particulares querrían tener su oro en moneda, acuñado en doblones, antes que en pasta, lo que favorecería al comercio. Con ello también se excusaría el inconveniente que se producía por la saca del oro en pasta que salía hacia España y de ahí a países extranjeros,

${ }^{15}$ Ordenanzas para la fábrica y valor de las monedas de oro y plata II Labrado, fundición, acunación y otras labores relacionadas con la fábrica de moneda, Biblioteca del Palacio Real, Madrid, Libro de las bulas y pragmáticas de los reyes católicos; impreso por L. Polono en Alcalá de Henares, 1503, tomo II, ed. facsímil, Madrid, 1973, XVII-XXI/225, ff. $199 \mathrm{v}-204 \mathrm{r}$. 
...por el ansia con le apetecen en pasta que ocassiona a que los mercaderes le estrabien para la utilidad que tienen de comprar con el las mercadurias y aunque no se escussa de todo punto esta negociacion tan perjudicial con labrar el oro por lo menos el que fuere a poder de estrangeros no tratan de conocido sin caussar los derechos que en beneficio del señoreage de la Cassa de la moneda.

Por todo ello, el virrey se veía en la obligación de hacer esta proposición, por el desvelo que en todo procuraba el mayor servicio de su majestad y el aumento de la Real Hacienda. Termina el documento con la siguiente cláusula de cortesía: "SMagd se servira de resolver y mandar lo que mas combenga de Dios", así como la data o fecha, "Mex 30 de marzo 653", y la firma como conde de Alba.

\section{La Real Gédula de 1655}

Una vez recibida la anterior proposición, se expidió una Real Cédula remitida al prior y a los cónsules de la Universidad de México de la Nueva España, transcrita al comienzo del siguiente apartado, el obedecimiento, con fecha tres de junio de $1655 .{ }^{16}$ Formalmente, cumple con las características de estas misivas en la época moderna en su concisión y esquematización gráfica y retórica. ${ }^{17}$ Comienza con un sucinto "El Rey" y las personas a quienes va dirigida, para pasar a relatar de modo escueto y concatenado el contenido de la petición anteriormente descrita y de la parte dispositiva, para terminar con una data tópica y la suscripción por parte del rey y por su mandato del secretario, en este caso Gregorio de Leguía.

Tras prácticamente transcribir la petición anteriormente vista, informa a los destinatarios que una vez analizada por el Real Consejo

${ }^{16}$ AGI, MÉXICO, 77, R.3, N.50 a.

${ }^{17}$ Riesco Terrero, Introducción a la paleografia y la diplomática general, p. 219. 
de Indias y nombrado fiscal en el asunto don Pedro de Gálvez, ordenaba que se juntasen los miembros del Consulado con ocho personas de las más antiguas en ese comercio, aunque no fuesen oficiales actuales, para que discurriesen sobre la materia y le informasen de las conveniencias e inconvenientes que pudiesen resultar de la introducción de esa nueva moneda de oro en esos reinos.

Los cálculos se podrían hacer, poco más o menos, por los libros de quintos, y se ordenaba que se estimase lo que supondría al año en aumento de ellos. Preguntaba, asimismo, sobre el perjuicio que podría suponer para las ocultaciones en el comercio de esas provincias con las Islas Filipinas, o si se estimaba que no se enviaba casi nunca, al ser necesario en este archipiélago el numerario en reales de plata en moneda corriente. Ordenaba también que se le informase del valor que debería darse a esa moneda, al poder ser un inconveniente hacerle corresponder con los 32 reales en los que se estimaba en los reinos de Castilla, por los 26 de su valor intrínseco. El monarca se interesaba también por la cantidad de señoreaje que se podría imponer sobre cada castellano, al tener menos coste la labra de 20 reales en oro que 20 en plata, y terminaba su Cédula con la indicación genérica de que se le remitiese información sobre cualquier otra conveniencia o inconveniente que se estimase, para que en el Consejo se proveyese lo que mejor conviniese al servicio y aumento de la Real Hacienda, de sus vasallos y del comercio.

\section{La contestación de Francisco de Montemayor}

Esta Real Cédula no fue contestada hasta casi ocho años después. Ante un escribano público, Matías Núñez, los miembros del Consulado acusaron recibo de la misma, y la recibieron de la siguiente manera:

...la tomaron y besaron y pussieron sobre sus cavezas y resolvieron que la obededesian y recivieron con la Reberencia y acatamiento debido como carta de su Rey y Señor Natural... 
Para darle cumplimiento acordaron que se le entregase a don Juan Francisco de Montemayor de Cuenca, del Consejo de su majestad, oidor de la Real Hacienda de Nueva España, juez de Apelaciones y ministro superior y al Consulado para que analizada se dispusiese lo que pareciese más conveniente para a la mayor brevedad se diese cumplimiento a lo que el rey ordenaba. Junto con el notario arriba indicado, constan como firmantes Tiburcio de Urrea, ${ }^{18}$ Cristóbal Jiménez y Fernando Cabeza de Vaca.

El fiscal estimó que al ser esta materia de suma gravedad se debían de juntar todos los papeles, y que se debía encargar a quien el Consulado estimase para que llamase al ensayador mayor del Reino y a los otros ensayadores, fundidores y balanzarios para tratar esa materia. En el expediente se encuentra, a continuación, el informe remitido por Francisco de Montemayor, junto con el parecer de Martín López, ensayador de México. ${ }^{19}$

Francisco de Montemayor informaba de que habiendo entrado en el turno de alzadas del Consulado de Nueva España había encontrado la Real Cédula de trece de junio de 1655, y como su cumplimiento no se había tratado, dispuso que se hiciese notoria del Consulado y que se pasase a las demás diligencias que constaban en el testimonio aportado. Se dispuso que el ensayador de la Casa de Moneda y el capitán don José de Quesada, perito en esas materias y apartador de oro de la Casa de México, hiciesen un escrito y con el mismo concurriesen a la Junta que se formaría para deliberar sobre todo y dar cuenta al monarca.

Según su testimonio, se hizo la junta, en la que reconoció poca gana en alguno de los interesados en el comercio del oro. Habiendo solicitado la última junta y conferencia, no había conseguido que se convocase, por lo que le pareció conveniente dar cuenta al monarca

${ }^{18}$ Probablemente hijo de Tiburcio de Urrea el viejo, mercader natural de Toledo y vecino de México, que falleció en el mar sin testar. Puede consultarse el expediente de los autos sobre sus bienes en el Archivo General de Indias, en adelante AGI, CONTRATACIÓN, 536, N.1, R.4.

${ }^{19}$ AGI, MÉXICO, 77, R.3, N.50 b. 
del informe del balanzario y ensayador Martín López, reconocido por persona perita en ese arte, para que sirviese como prueba de lo conveniente que sería labrar doblones y escudos en el Reino de Nueva España, tanto para el monarca como para sus vasallos, "....aunque a costa, y sentimiento, de algunos particulares que tienen su granxeria, en el comercio y extrassion del Oro". Por su parte, estimaba que la labra de moneda sería el medio más a propósito y eficaz para llevar el dinero a España, y ese parecer le fue comunicado al virrey.

\section{El informe de Martín López}

Este informe está fechado el día 2 de junio de 1663. Comienza afirmando, por contraste indubitable, la conveniencia y utilidad al monarca y a sus vasallos de que de todo el oro que se sacase del reino cobrase el soberano el señoreaje, que podía ser de mucha entidad, haciéndose el cómputo respectivamente con la plata, dado que en esta última se daba al monarca un real en cada marco, comprendiendo cada marco de oro doce reales de plata y seis tomines y granos. Si se reducía el oro a moneda, llevaría ya deducida la utilidad y ganancia del señoreaje, con lo que se conseguía el comercio en los reinos con esa moneda, y asimismo se evitaba la saca que se producía en pasta. ${ }^{20}$

En cuanto a la posible inconveniencia de su labra para el comercio con Filipinas, juzgaba que no lo había, dado que en el archipiélago no necesitaban de esa moneda, y todo el comercio consistía en moneda de plata. Afirmaba también que para tener conocimiento de la utilidad y los derechos a cobrar por su labra se debería pedir razón a los reinos del Perú, donde la moneda de oro ya se había labrado, para que en Nueva España se asignase más o menos lo mismo o que su majestad asignase los derechos de señoreaje, las costas de la labor y la ganancia de los mercaderes que le pareciere.

Repasa el ensayador el valor del oro y de los derechos que quedaban en la legislación vigente en ese momento. Cita en primer lugar la Ley II, título 21, libro $5^{\circ}$ de la Pragmática de 14 de diciembre de 1612, por

${ }^{20}$ AGI, MÉXICO, 77, R.3, N.50 c. 
la que el castellano de oro de ley de 22 quilates quedó valorado en 16 reales y 32 maravedíes. Hace referencia asimismo a la Ley 13, título 21, libro 5 de la Nueva Recopilación, en la que se ordenaba que se labrase moneda de oro en escudos sencillos y dobles de oro de 22 quilates y ley de sesenta piezas de escudos dobles y sencillos por marco. También cita la Ley 16, título 54, libro 5º, promulgada en 1609 de la Novísima Recopilación, que mandó que el escudo sencillo valiese 440 maravedíes. Con base en toda esta normativa, el ensayador concluía que un doblón de a dos tenía un peso de 141 granos y 3/15 de grano, o un castellano tres tomines nueve gramos y $3 / 17$ de grano de ley de 22 quilates, $y$ un valor de 845 maravedíes y 1/15 de maravedí en pasta sin labrar, y hecho moneda 880 maravedíes, con lo que quedaría en concepto de señoreaje por diferencia la cantidad de 32 maravedíes y 16/15 de maravedí en cada doblón de a dos, por lo que en cada marco de oro de 22 quilates de 50 castellanos del que salían 68 piezas en escudos sencillos y doblones quedarían para costas, señoreaje y ganancia del mercader 1120 maravedíes, o 32 reales y 32 maravedíes.

Si por cada castellano labrado comprado a su valor en plata correspondía como señoreaje ocho maravedíes y 11/50 de maravedí, y si se estimaba que se podrían labrar en esos reinos poco más o menos 53693 marcos de oro al año, correspondería al monarca en concepto del $1 \frac{1}{2} \%$ y quinto 15623 marcos, y quedarían en el comercio 58090 marcos. Y si bien parte de esta cantidad se utilizaría para labrar joyas o batirlo en panecillos para dorar, el ensayador estimaba que no pasaría de 50 marcos. Todos estos cálculos los hizo con base en el señoreaje en cada castellano de oro del cero 11/20 maravedíes por marco, el regulado para la plata, habiendo ya sacado los 20 reales y 29 maravedíes en cada marco de oro. Todo ello se vería incrementado por las alcabalas y otros tributos al comercio, que no sería poco, al entrar en el comercio más de 116000 pesos.

El ensayador afirmaba igualmente que, además de los beneficios para el monarca, al labrarse el oro se volvía su valor en moneda y quedaba en su intrínseco valor de 26 reales menos cuatro maravedíes. En cuanto a si debería correr en esos reinos a los 32 reales que lo hacía en España el doblón de a dos, 
...su Magestad como dueño de todo mandara lo que fuere seuido, y aunque no se labre mas que la cantidad arriba expressada es mucho el util que gozara su Mag y todos sus vassallos y este es mi parezer.

\section{La Junta del Consulado de Comercio}

Tras transcribir la Real Cédula de 1655, ${ }^{21}$ comienza la relación de las actuaciones realizadas para su cumplimiento, siendo la primera fecha citada el 19 de mayo de 1663, cuando Juan Francisco de Montemayor informa que se debían elegir con toda presunción y brevedad las personas que se considerasen más inteligentes en la materia y señalar el día para que se juntasen en la casa del Consulado, para dar noticia al excelentísimo señor marqués conde de Baños, virrey de Nueva España. En toda la documentación transcrita consta la presencia de Matías Núñez, escribano real. ${ }^{22}$

En fecha 21 de mayo se informaba por el prior y los cónsules de que se había designado para tal fin:

...al Contador Phelipe Nauarro Y Latiença Capitanes Domingo de Cantabrana, Pedrosedano Esteuan de Molinamosquera, Alonsso de Valdes, Josep dequesada, JosephdeEretes Mercaderes de platta Y encomenderos Y a Geronimo bezerra Ensayador de las Rl Cassa de lamoneda de este reino.

Y se ordenó que se les llamase por papel de convocatoria junto al prior y los cónsules, el miércoles 23 del mes corriente a las nueve de la mañana en la casa de dicho Consulado. Nuevamente se repite la fórmula antes aludida de acatamiento, tomándola y besándola y poniéndola sobre sus cabezas. Junto al nombre del escribano Matías Núñez aparecen también los de Tiburcio de Urrea, Cristóbal Jiménez

\footnotetext{
${ }^{21}$ AGI, MÉXICO, 77, R.3, N.50 d.

${ }^{22}$ AGI, MÉXICO, 77, R.3, N.50 e.
} 
y Fernando Cabeza de Vaca. Consta, asimismo, en el expediente la transcripción del papel de convocatoria, de la misma fecha, y el llamamiento y la fe registral del notario de su recibimiento.

Una nueva convocatoria se fijó para el día 9 de junio de 1663, sábado, a las tres de la tarde. El portero del Consulado, Sebastián de Aguilera, hizo el llamamiento de los intervinientes, dando fe de ello el notario y los testigos Felipe González y Antonio Díaz. Los intervinientes afirmaron en cuanto al primer punto que de los libros de las Cajas Reales de un año con otro se podía saber la cantidad de oro a acuñar, que estimaban entre 120000 a 130000 pesos poco más o menos, y que de esta cantidad más de una cuarta parte se consumía en la labra de cadenas y panecillos para dorar bordaduras y otras cosas que se fabricaban en el reino, por lo que quedarían para acuñar de 90 a 100000 pesos.

En cuanto al valor que se podría dar a la moneda, entendían que éste, en todas las monedas que se labraban en los reinos de la monarquía, debía ser y era del mismo valor, lo que no se podía alterar por ninguna razón, si no fuese por alguna lógica y motivo que ese Consulado no podía comprender, al faltarle noticias de la forma en que se labraba la moneda en las Casas de Moneda de Castilla. Por ello, el Consulado solicitaba que se demandase a los tesoreros de las mismas que remitiesen relación de la forma en la que se labraba la moneda con las siguientes circunstancias:

- Los quilates de ley de esa moneda.

- Los escudos que se sacaban de cada marco.

- El valor y peso que debían tener los escudos.

- Los derechos que se pagaban en la Casa de Moneda de monedaje y demás costas, haciendo cómputo por cada marco.

- $\quad$ El señoreaje y en qué forma se pagaba a su majestad de cada marco.

Una vez conocido lo anterior, el Consulado podría reconocer la utilidad que al monarca y al comercio podría venir de labrar moneda en el reino. 
En el tercero de los puntos a tratar, estimaban que para que el monarca tuviese aumentos en su Real Hacienda siempre se debía buscar alguna a sus vasallos y comercio, dado que el mercader que tenía oro en pasta y que ya tenía pagados al soberano sus reales quintos no se le podía obligar a que lo labrase en moneda, como sucedía con la plata. Como habían reconocido los reyes católicos y Carlos v, se les concedió a todos los que descubriesen minas y tesoros en el reino toda la plata que sacasen, siendo patrimonio del monarca, pagando los quintos reales, reconociéndose, con ello, la gran utilidad y los grandes aumentos tenidos por los soberanos.

José de Vetes, habiendo visto y leído la notoria y resolución de la Junta, afirmó que estaba de acuerdo con lo resuelto y que era del mismo sentir y parecer. Esteban de Molina afirmó que no pudo asistir a la junta por estar achacoso, y que estaba conforme con lo resuelto. Alfonso Valdés, también enfermo, no pudo asistir, pero estaba asimismo de acuerdo con lo resuelto. En el mismo sentido se manifestó el capitán Domingo de Cantabrana. De todo ello levantó fe el notario Matías Núñez.

El día 11 de junio, el escribano dio noticia a Juan Francisco de Montemayor de lo resuelto en la junta, que dictaminó que no se fuese la Flota sin dar noticia de lo resuelto al monarca. Esta pieza concluye con las formalidades al uso, dando fe de que todo ello había sido sacado, corregido y concertado con su original y diligencias realizadas hasta esa fecha, cuyo original quedaba en el archivo del Consulado, y la fe de que Matías Núñez era escribano real y del Consulado.

\section{La recepción del expediente por el Consejo de Indias}

En fecha 11 de febrero de 1664, en un papel sellado de un cuartillo, consta la recepción de las actuaciones llevadas a cabo y un resumen de las mismas. ${ }^{23}$ Una vez vistas en el Consejo, se remitió al fiscal, que estimó que la materia era de suma gravedad, y pidió que se juntasen todos los papeles que hubiese, se sometiesen a quien el Consejo estimase y que se llamase al ensayador mayor del Reino, y a los otros ensayadores,

${ }^{23}$ AGI, MÉXICO, 77, R.3, N.50 f. 
fundidores y balanzarios y con ellos se practicase esta materia y el parecer del ensayador Martín López, para que a la vista de todo y de los informes se tomase la resolución que más conviniese. Este resumen se despachó en Madrid el 5 de marzo de 1664, al licenciado Navarro, y la carta para la Casa pidiendo el informe se remitió el día 4 de noviembre de $1664 .{ }^{24}$

\section{Conclusión}

El 25 de febrero de 1675, ya en el reinado de Carlos II, se autorizó la acuñación en oro, y las primeras monedas batidas en este metal lo fueron en México, a finales del siglo en Lima y en 1698 en Potosí. Vázquez Pando afirma que la ley y peso de las monedas de oro fue fijada por el virrey, fray Payo Hernández de Rivera, en 22 quilates y 68 escudos por marco, la ley y peso fijados desde 1537 para los escudos, y que las acuñaciones comenzaron en diciembre de $1679,{ }^{25}$ si bien Craig recoge que en algunos catálogos se han publicado atribuciones de monedas de oro de esta ceca anteriores a 1697, ${ }^{26}$ y Pradeau especulaba que podría ser cierto, si bien él no había visto ninguna. ${ }^{27}$

También se autorizó la emisión de moneda de oro en Cuzco, en 1683. ${ }^{28}$ Para la ceca de México se previó la emisión de piezas de ocho, cuatro, dos y un escudo, con una ley de 22 quilates y una talla de 68 escudos el marco. Las primeras emisiones, medias onzas, presentan en su anverso el escudo grande de la monarquía, y en su reverso la cruz de Jerusalén, como en la Península, y son de una gran tosquedad.

${ }^{24}$ AGI, MÉXICO, 77, R.3, N.50 g.

${ }^{25}$ Vázquez Pando, "Algunas observaciones sobre el derecho monetario de la Nueva España”, pp. 1686-1687.

${ }^{26}$ Craig, Spanish colonial gold coins in the Florida Collection, p. 36.

${ }^{27}$ Pradeau, Numismatic History of Mexico, p. 46.

${ }^{28}$ Craig, Spanish Colonial Gold Coins in the Florida Collection, pp. 31-32, citando a Cañete y Domínguez, hace referencia a un despacho de 6 de enero de 1683 y a una Real Cédula de 26 de febrero del año siguiente. Asimismo, recoge que de acuerdo con Medina una carta del marqués de la Monclova a Carlos II, del 22 de diciembre de 1697 sugiere que esta ceca seguía abierta y lista para seguir acuñando moneda de oro. Craig estima que la práctica totalidad de la producción de moneda áurea de esta ceca de 1698 se hundió con la Flota de 1715. 


\section{Fuentes}

\section{Archivos}

AGI Archivo General de Indias

\section{Bibliografía}

Beltrán Martínez, Antonio, Introducción a la numismática universal, Madrid, Istmo, 1987.

Carson Brevoort, James, Early Spanish \& Portuguese Coinage in America, Boston, T.R. Marvin \& Son, 1885.

Céspedes del Castillo, Guillermo, "Las cecas indianas en 1536-1825", en Anes y Álvarez de Castrillón, Gonzalo, y Céspedes del Castillo, Guillermo, en Las Casas de Moneda en los Reinos de Indias, vol. I, Madrid, FNMT, 1996.

Chacón, Néstor Ricardo, Derecho Monetario, Bogotá, Librería Ediciones del Profesional, 2005.

Craig, Alan K., Spanish Colonial Silver Coins in the Florida Collection, Gainesville, University Press of Florida, 2000.

, Spanish Colonial Gold Coins in the Florida Collection, Gainesville, University Press of Florida, 2000.

Fonseca, Fabián de, y Carlos de Urrutia, Historia general de la Real Hacienda, por orden del virrey conde de Revillagigedo, t. I, México, Imprenta de Vicente García Torres, 1845.

Gil Farrés, Octavio, Historia de la moneda española, Madrid, Gráficas Adrados, 1976. 
Menzel, Sevall, Cobs, Pieces of Eight and Treasure Coins, The American Numismatic Society, Nueva York, 2004.

Moretti, conde de, Manual alfabético razonado de las monedas, pesos y medidas de todos los tiempos y países, con las equivalencias españolas y francesas, Madrid, Imprenta Real, 1828.

Pradeau, Alberto Francisco, Numismatic History of Mexico from the Pre Columbian Epoch to 1823, Los Ángeles, Western Printing Company, 1938.

Riesco Terrero, Ángel, Introducción a la paleografia y la diplomática general, Madrid, Síntesis, 2000.

Vázquez Pando, Fernando Alejandro, "Algunas observaciones sobre el derecho monetario de la Nueva España", en Memoria del x Congreso del Instituto Internacional de Historia del Derecho Indiano, 1995, pp. 1675-1706.

Villaplana Persiva, Manuel, Historia del Real de a Ocho, Murcia, Universidad de Murcia, 1997. 